\title{
Method for Detection and Stereoscopic Measuring Lane Markers
}

\author{
C. Y. Mu, X. Ma, C. T. Zhang \\ Institute of Information and Communication Technology \\ Beifang University of Nationalities \\ Yinchuan, P.R. China
}

\begin{abstract}
A method for detection and stereoscopic measuring lane markers for safety driver assistance was proposed. Firstly, it was concerned on selecting an appropriate limited region of interest by OTSU segmentation, which would be candidate areas for lane boundaries detection by Sobel and piecewise fitting. Results of lane detection were combined with calibrated camera parameters for measuring distance between lane and ego car. Experiments show that lane markers can be detected correctly although some situations are fade. In order to detecting and measuring lane markers more precisely, several characteristics of test environments need to be considered in further.
\end{abstract}

Keywords-stereoscopic measuring; lane detection; OTSU; calibrated camera parameters

\section{INTRODUCTION}

\section{A. Background}

Majority of traffic accidents are caused by error operations or distractions according to the traffic departments' statistics. Accidents caused by these cases could be avoided by continually monitor the position of a car in lanes.

It has been an active field of research for the past two decades, and many considerable progress been made. Some modern series-production vehicles have been already equipped with monocular vision system, e.g. blind pot monitoring, etc. Compared with monocular image, it is particularly important that we could understand a traffic situation from binocular imagery. While this additional information could open new application areas, gathering and analyzing it is challenging.

\section{B. Current Methods}

Previous research has been conducted on modeling the road profile from the disparity maps received through binocular vision and stereo matching, because it is the basic step of understanding the surrounding environment of a vehicle. By comparative performance, $\mathrm{BP}$ is a good choice if there is insignificant variation of depth in the imagery. GC can handle the problems of ordering constraint, but failed to mask-image, for example windscreen wiper is moving [1-2].

In research of Nedvschi and Danescu, a real-time environment representation based on occupancy grid temporal analysis using a dense stereo-vision system is proposed [3]. Stereo imaging can be used for the same basic tasks as LIDAR [4], including obstacle identification, host to road pitch angle estimation, curb detection and 3D road geometry and slope estimation [5].

\section{Proposed Method}

This paper proposes method detection and stereoscopic measuring lane markers. It is consists of four parts: a. image transformation, b. region of interest setting by OSTU segmentation, c. lane markers detection by Sobel and piecewise fitting, d. measurement of lane markers by stereoscopic method with calibrated parameters, fig. 1 .

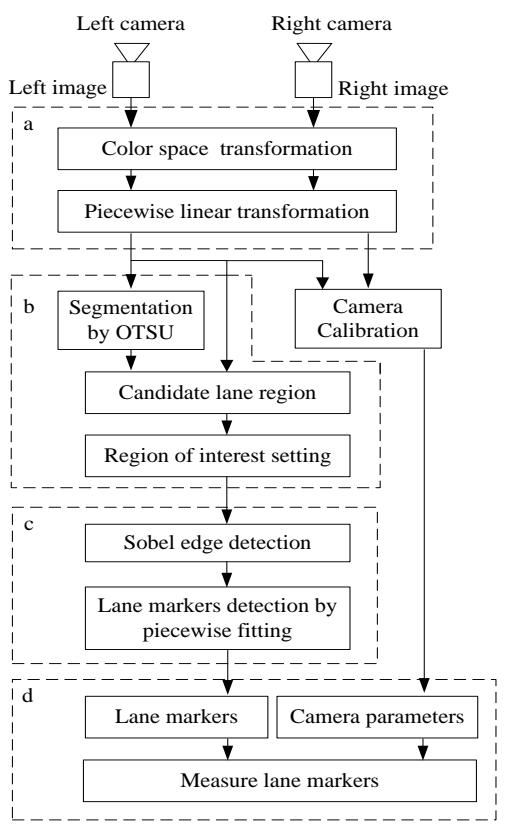

FIGURE I. FLOW CHART OF THE ALGORITHM.

Rest of this paper is organized as follows. Section 2 describes performance of lane detection and measurement algorithm. Several experimental results are presented to support the validity of this method in section 3 , and section 4 concludes the paper.

\section{ALGORITHM}

\section{A. Image Transformation}

1) Color space transformation: The most visually significant information in the colour image is reserved in $\mathrm{Y}$ component of image. So $\mathrm{Y}$ component of image is used to detect edges of desired lane.

2) Piecewise linear transformation: It's necessary to transform an image when the enviroment is dim or the image 
has low contrast. Piecewise linear transformation (PLT) is adopted in this method. We choose the minimum and maximum input gray value as segement points. Fig. 2 (a) and (b) are an original image and the PTL-based image, respectively. The original image has a low contrast, whose detail cannot see clearly. The PTL-based performent result has been enhanced.

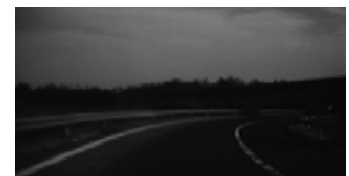

(a) Original image

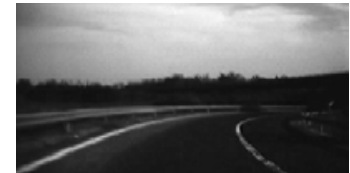

(b) PLT-based image
FIGURE II. RESULTS OF PIECEWISE LINEAR TRANSFORMATION.

\section{B. Region of Interest Setting}

1) OTSU segmentation: OTSU segmentation algorithm was proposed by Japanese researcher Nobuyuki Otsu in 1979. The criterion is that maximize the separabilitiy of the resultant classes in gray levels [6]. For extended, $m-1$ discrete thresholds can divide image into $m$ classes. In this approach, we use two thresholds: $1 \leq k_{1}<k_{2}<L$ for separating three classes, $C_{0}$ for $\left[1, \ldots, k_{k 1}\right],{ }_{C 1}$ for $\left[k_{1}+1, \ldots, k_{2}\right]$, and ${ }_{C 2}$ for $\left[k_{2}+1, \ldots, L\right]$. Then the probabilities of class occurrence, and the class mean levels are: $\omega_{0}, \omega_{1}, \omega_{2}, \mu_{0}, \mu_{1}, \mu_{2}$. And the total mean level of the original picture is $\mu_{T}$, and there is a relation for choice of ${ }_{k 1}$ and ${ }_{k 2}$,

$$
\omega_{0} \mu_{0}+\omega_{1} \mu_{1}+\omega_{2} \mu_{2}=\mu_{T}, \quad \omega_{0}+\omega_{1}+\omega_{2}=1
$$

The class variances are given by $\sigma_{0}^{2}, \sigma_{1}^{2}, \sigma_{2}^{2}$. The criterion measure $\sigma_{B}^{2}$ is then a function of two variables and an optimal set of thresholds $k_{1}^{*}$ and $k_{2}^{*}$ is selected by maximizing $\sigma_{B}^{2}$ :

$$
\sigma_{B}^{2}\left(k_{1}^{*}, k_{2}^{*}\right)=\max _{1 \leq k_{1}<k_{2}<L} \sigma_{B}^{2}\left(k_{1}, k_{2}\right)
$$

Results of object segmentation by OTSU are shown in fig.3. This test scene is divided into three classifications. And they are represented by three constants, e.g. 0, 0.498 and 1 .

The most prominent of three classifications is that the lane markers, road and background can be divided hierarchically. Most of the lane markers could be detected, but the result of lane detection still have some breakpoint. Morphological operate can enchance the performance of classification result disturbed by noisy or shadow.

2) Appropriate limited region of interest: Lane images are captured by a camera which can be situated inside a car near rear view mirror. In upper part of these lane images are sky, buildings, flyovers, trees, street lamps, hills, etc. They have solid liner structure which could be detected by edge operator. And they might disturb the detection of candidates of lane markers in lower part of images. Thus, it is necessary to set lower area of lane image as ROI. An appropriate limited region of interest (ALROI) is obtained by combined the PLT- based image $\mathrm{b}(\mathrm{x}, \mathrm{y})$ with ROI and the model $\mathrm{Z}(\mathrm{x}, \mathrm{y})$, which $\mathrm{x}$ and $y$ are coordinate axes in image.

$$
\operatorname{ALROI}(x, y)=b(x, y) \cdot \operatorname{ROI}(x, y) \cdot\left(Z(x, y)>=T h_{a}\right)
$$

Where, $T h_{a}$ is a threshold for selecting candidate areas from the PLT-based image. The limited ROI helps to give lane identification in an appropriate region. This process will have the effect of enhancement in the speed of operation.

Candidate lane regions are shown in fig. 4, ALROI covered the majority lane markers, and most of their surrounding environment was masked.

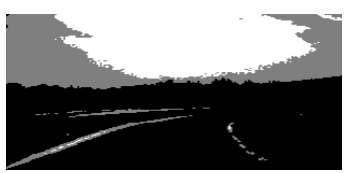

FIGURE III. CLASSIFICATION RESULT.

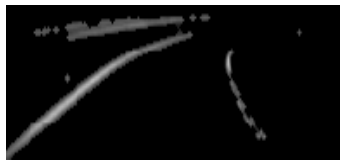

FIGURE IV. CANDIDATE LANE REGION.

\section{Lane Markers Detection}

1) Sobel edge operator: Edges are important features in an image since they represent significant local intensity changes and offer vital clues to separate regions within an object or to identify changes in illumination. There are many ways to perform edge detection, Roberts, Canny, Prewitt and so on. We used Sobel edge detection in the limited region.

2) Piecewise Lane Marker Fitting: Piecewise lane marker fitting is widely used in a roadway under a variety of complicated conditions [7]. In this method, smooth piecewise polynomial functions are used in representing lane markers.

$$
\text { Lane }(x, y)= \begin{cases}a x^{2}+b x+c, & \text { if } x>x_{m} \\ d x+e, & \text { if } x \leq x_{m}\end{cases}
$$

Where, $x_{m}$ represents the border between near and far fields. The linear part of the model is used to fit the near vision field, while the parabolic model fits the far field. This proposed technique is robust in the presence of noise, shadows, lack of lane painting and change of illumination conditions.

Results of lane detection are pointed out in the gray image with yellow lines in fig. 5. Lane markers can be detected correctly although the environment are complicated. The intensity of image in fig. 5 (a) is dim, performance of detection has been enhanced by PLT-based. Piecewise lane marker fitting could counteract this disturb. And one of the lane markers is a dotted line in fig. 5(b), detection results is also correct. 


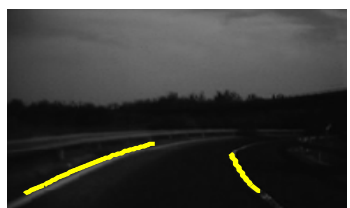

(a) Scene 1

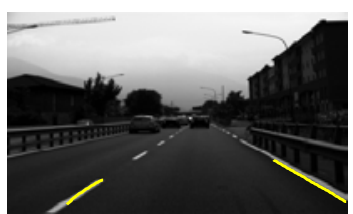

(b) Scene 2
FIGURE V. RESULTS OF LANE MARKERS DETECTION.

\section{Measurement of lane markers}

1) Calibration board: The board used in this calibration process contains 11 by 9 circle blobs. And there are five bigger in them, called signed blobs. They are located firstly and then others could be derived through them. This calibration board are designed by taking advantage of fitting for accuracy in computation.

2) Transform between coordinates: In fact, there is a transform between real world coordinate system and image. As:

$$
Z_{\mathrm{c}}\left[\begin{array}{l}
u \\
v \\
1
\end{array}\right]=\left[\begin{array}{ccc}
\alpha_{\mathrm{x}} & 0 & u_{0} \\
0 & \alpha_{\mathrm{y}} & v_{0} \\
0 & 0 & 1
\end{array}\right]\left[\begin{array}{ll}
R & t
\end{array}\right]\left[\begin{array}{l}
x_{w} \\
y_{w} \\
Z_{w} \\
1
\end{array}\right]
$$

Where, $\left(x_{w}, y_{w}, z_{w}\right)$ means the world coordinate of an object, $a_{x}, a_{y}, u_{0}$ and $v_{0}$ are the inner parameters of stereoscopic vision system, $a_{x}$ and $a_{y}$ mean the ratio of focal length $f$ in $u$ and $v$ direction axis respectively, $u_{0}$ and $v_{0}$ mean the point which focal axis crossed with the image, $\mathrm{R}$ and $\mathrm{t}$ are the outer parameters of this system. They are rotation and translation matrix. Eqn. 6 could be further derived through mathematical deduction.

$$
\left[\begin{array}{l}
x_{w} \\
y_{w} \\
z_{w}
\end{array}\right]=\left(\mathrm{M}^{\prime} \times \mathrm{M}\right)^{-1} \times M^{\prime} \times U
$$

Where, matrix $\mathrm{M}$ and $\mathrm{U}$ are consisted with inner or outer parameters of the stereoscopic vision system.

We could get an object coordinate in world by calculated with these calibrated parameters. Moreover, distance between any two points could be measured by calculating the locating in real world coordinate system.

\section{EXPERIMENTAL RESULTS}

\section{A. Measurement in Indoor Environment}

We have tested our method on measurement a stand of lighter in indoor environment. Fig. 6 is shown the real stand and its schematic drawing. Table. 1 gives the measured values and the calculated values.

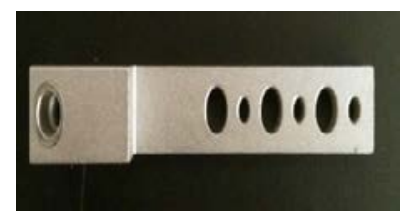

(a)a stand of lighter

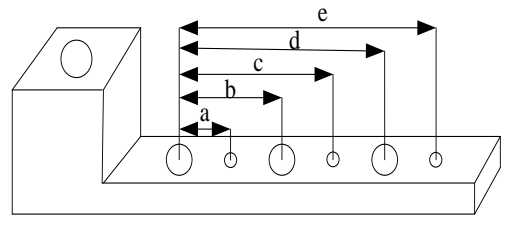

(b) the schematic drawing of a stand of lighter FIGURE VI. MEASUREMENT OF A STAND OF LIGHTER.

TABLE I. MEASUREMENT RESULT OF A STAND OF LIGHT.

\begin{tabular}{|c|c|c|c|c|}
\hline \multirow{2}{*}{} & $\begin{array}{c}\text { Calculated } \\
\text { values (mm) }\end{array}$ & $\begin{array}{c}\text { Measured } \\
\text { values } \\
\text { (mm) }\end{array}$ & $\begin{array}{c}\text { Absolute } \\
\text { error (mm) }\end{array}$ & $\begin{array}{c}\text { Relative } \\
\text { error } \\
\mathbf{( \% )}\end{array}$ \\
\hline \multirow{2}{*}{ a } & 8.04 & 8.07 & 0.03 & 0.37 \\
\cline { 2 - 5 } & 8.02 & 8.06 & 0.04 & 0.50 \\
\hline \multirow{2}{*}{ b } & 15.99 & 16.02 & 0.03 & 0.19 \\
\cline { 2 - 5 } & 15.96 & 16.00 & 0.04 & 0.25 \\
\hline \multirow{2}{*}{ c } & 24.09 & 24.07 & 0.02 & 0.08 \\
\cline { 2 - 5 } & 24.06 & 24.05 & 0.01 & 0.04 \\
\hline \multirow{2}{*}{ d } & 32.12 & 32.11 & 0.01 & 0.06 \\
\cline { 2 - 5 } & 32.11 & 32.10 & 0.01 & 0.03 \\
\hline \multirow{2}{*}{ e } & 40.07 & 40.05 & 0.02 & 0.05 \\
\cline { 2 - 5 } & 40.11 & 40.10 & 0.01 & 0.02 \\
\hline
\end{tabular}

From above, the absolute error between calculated values and measured are smaller than $0.05 \mathrm{~mm}$. And the relative error is also very small, especially in test c, $\mathrm{d}$ and $\mathrm{e}$.

\section{B. Measurement of Lane Markers}

In order to test our measurement on lane markers, we have put stereoscopic cameras on a tripod on test road for capturing stereo images, calibrating cameras, estimating the distance between signed points and cameras. Stereoscopic test on lane markers is shown in fig. 7.

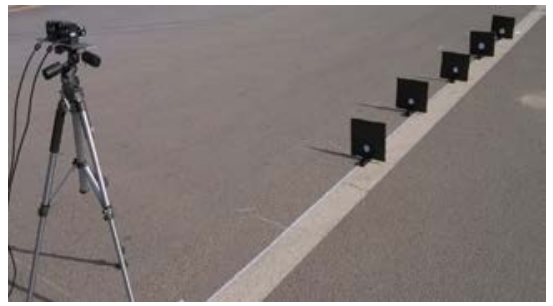

FIGURE VII. STEREOSCOPIC TEST ON LANE MARKERS.

Then the lane detection and measurement algorithm are simulated in MATLAB. We can get serveral coordinate of identified points in left and right image separately. By coordinate transfrom, these points coordinate in real world 
could be calculated either. The measured diatance and calculated by stereo theories are listed in table. 2 .

TABLE II. RESULTS OF MEASUREMENT ON LANE MARKERS.

\begin{tabular}{|c|c|c|c|c|}
\hline \multirow{2}{*}{ a } & $\begin{array}{c}\text { Calculated } \\
\text { value (m) }\end{array}$ & $\begin{array}{c}\text { Measured } \\
\text { value (m) }\end{array}$ & $\begin{array}{c}\text { Absolute } \\
\text { error (m) }\end{array}$ & $\begin{array}{c}\text { Relative } \\
\text { error (\%) }\end{array}$ \\
\cline { 2 - 5 } & 10.262 & 10.0 & 0.262 & 2.62 \\
\hline \multirow{2}{*}{ b } & 10.367 & 10.0 & 0.367 & 3.67 \\
\cline { 2 - 5 } & 20.513 & 20.0 & 0.513 & 2.57 \\
\hline \multirow{2}{*}{ c } & 31.068 & 30.0 & 1.068 & 3.56 \\
\cline { 2 - 5 } & 31.232 & 30.0 & 1.232 & 4.11 \\
\hline
\end{tabular}

Relative error between measured and calculated values are bigger than the test result in indoor environment. There are many infactors influence of this test results. In this stereoscopic camera system, the identified points setting are signed on boards in vertical plane, which is not close to the ground. And the sigened points in far sight are occupied smaller number of pixels in image. So the pixel of far sight has much more influence than nearby sight situations. A minimal error or deviation may result in wide divergence.

\section{CONCLUSIONS}

In this paper, a method for detection and measurement lane markers is presented. This study was concerned on selecting an appropriate limited region of interest by OTSU segmentation, generating candidates for lane markings and finally finding the lane markers by Sobel and piecewise fitting. Then combined lane detection results with calibrated parameters, we could get measured distance between lanes and car. We test our experiments in indoor environment firstly, and the result is well. Then we have experiments in outdoor, results shown that lane boundaries could be detected correctly although some situation was fade, but the relative error of measured distance between lanes and ego car is not always perfect. In order to detect and measure lane markers more precisely, several characteristics of environment should be considered in further.

\section{ACKNOWLEDGEMENTS}

This work was financially supported by the National Natural Science Foundation of China (61162005 and 61163002), Natural Science Foundation of Ningxia Province (NZ14107), Open Foundation of State Key Laboratory of Robotics and System (SKLRS-2013-MS-05).

\section{REFERENCES}

[1] Mahmood, F., Haider, S., \& Kunwar, F., Investigating the performance of Correspondence Algorithms in Vision based Driver-assistance in Indoor Environment. International Journal of Computer Applications, 60(9), pp. 6-12, 2013.

[2] Schauwecker, K., Morales, S., Hermann, S., \& Klette, R., A comparative study of stereo-matching algorithms for road-modeling in the presence of windscreen wipers. In Intelligent Vehicles Symposium (IV), pp. 7-12, 2011.
[3] Danescu, R., \& Nedevschi, S., New results in stereovision based lane tracking. In Intelligent Vehicles Symposium (IV), pp. 230-235, 2011.

[4] Chen, L., Li, Q., Li, M., Zhang, L., \& Mao, Q., Design of a multi-sensor cooperation travel environment perception system for autonomous vehicle. Sensors, 12(9), pp. 12386-12404, 2012.

[5] Hillel, A. B., Lerner, R., Levi, D., \& Raz, G., Recent progress in road and lane detection: a survey. Machine vision and applications. 25(3), pp. 727-745, 2014.

[6] Otsu N., A threshold selection method from gray-level histograms. Automatica, 9(1): pp. 62-66, 1975.

[7] Jung, C. R., Kelber, C. R., A robust linear-parabolic model for lane following, IEEE 17th Brazilian Symposium on Computer Graphics and Image Processing. pp. 72-79, 2004. 